\title{
Diagnostic Evaluation of Infants with Recurrent or Persistent Wheezing
}

\author{
Debra Boyer ${ }^{1}$, Emily Barsky ${ }^{1}$, Christina Michelle Papantonakis ${ }^{2}$, Jessica Pittman ${ }^{3}$, Clement L. Ren ${ }^{4}$, \\ Charles R. Esther Jr. ${ }^{5}$, Kevin C. Wilson ${ }^{6}$, and Carey C. Thomson ${ }^{7}$ \\ ${ }^{1}$ Boston Children's Hospital, Harvard Medical School, Boston, Massachusetts; ${ }^{2}$ Vanderbilt University School of Medicine, Nashville, \\ Tennessee; ${ }^{3}$ Washington University School of Medicine, St. Louis, Missouri; ${ }^{4}$ Indiana University School of Medicine, Indianapolis, \\ Indiana; ${ }^{5}$ University of North Carolina School of Medicine, Chapel Hill, North Carolina; ${ }^{6}$ Boston University School of Medicine, Boston, \\ Massachusetts; and ${ }^{7} \mathrm{Mt}$. Auburn Hospital, Harvard Medical School, Boston, Massachusetts
}

Official ATS clinical practice guidelines: Diagnostic Evaluation of Infants With Recurrent or Persistent Wheezing. Am J Respir Crit Care Med 2016;194:356-373.

The Pediatric Assembly of the American Thoracic Society assembled an interdisciplinary panel to develop clinical practice guidelines for the evaluation of infants with recurrent or persistent wheezing. This summary of the guideline is intended for practicing clinicians.

Wheezing during infancy is common: a longitudinal birth cohort study of healthy, full-term infants found that $34 \%$ of these children had at least one episode of wheezing before age 3 years (1). Although many of these children have mild, easily treated wheezing, some progress to have persistent wheezing that does not respond to conventional therapies.

For the purposes of these guidelines, infantile wheezing is defined as recurrent or persistent episodes of wheezing in infants younger than 24 months of age. The guidelines address infantile wheezing that is persistent despite treatment with bronchodilators, inhaled corticosteroids, or systemic corticosteroids.

Recommendation 1: For infants with persistent wheezing despite treatment with bronchodilators, inhaled corticosteroids, or systemic corticosteroids, we suggest airway survey via flexible fiberoptic bronchoscopy (conditional recommendation, very low quality of evidence).

Ten case series that enrolled 1,364 children were identified. The case series reported that $33 \%$ of children who underwent airway survey for respiratory symptoms were found to have an anatomic abnormality known to cause wheezing. The most common lesions were airway malacia and compression by vascular structures.

Infants with airway malacia are usually managed by observation alone, because the vast majority improve over time. Interventions such as positive pressure ventilation, surgery, or stenting are reserved for infants with life-threatening complications, such as complete airway obstruction, respiratory failure, recurrent pneumonia, or failure to thrive.

Aortopexy is the most common surgical intervention; it relieves airway obstruction in nearly all patients with tracheomalacia but is less effective in patients with tracheobronchomalacia. Airway stenting may also improve airway obstruction, but the formation of granulation tissue is a common and problematic complication. Vascular anomalies, such as vascular rings and vascular slings, are unlikely to self-resolve; therefore, surgical intervention is usually performed in symptomatic patients. In seven case series, improvement in symptoms occurred in 88 to $100 \%$ of patients, with complete resolution achieved in $50 \%$ of patients.

Taken together, the evidence suggests that approximately $30 \%$ of infants with persistent wheezing who undergo a bronchoscopic airway survey are likely to benefit from the evaluation. Benefits include identifying a lesion that may be surgically corrected; avoiding the burden, costs, and potential harms of further diagnostic testing; reducing the use of ineffective medications; reducing the frequency of physician visits; and providing parental reassurance. These benefits were judged by the guideline committee to outweigh the burdens, cost, and complications of flexible bronchoscopy.

Recommendation 2: For infants with persistent wheezing despite treatment with bronchodilators, inhaled corticosteroids, or systemic corticosteroids, we suggest bronchoalveolar lavage (BAL) (conditional recommendation, very low quality of evidence).

Twenty case series were evaluated and found that, on average, 40 to $60 \%$ of infants with recurrent wheezing had a positive BAL culture. A randomized trial of children with cough due to presumed bacterial bronchitis found that $48 \%$ of children who received

(Received in original form July 27, 2016; accepted in final form July 29, 2016)

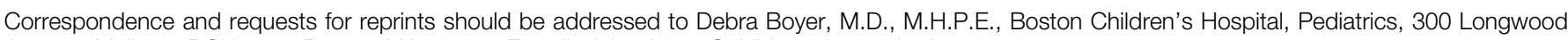
Avenue, Mailstop BCH3121, Boston MA 02115. E-mail: debra.boyer@childrens.harvard.edu

CME will be available for this article at www.atsjournals.org

A Maintenance of Certification exercise linked to this summary is available at http://www.atsjournals.org/page/ats_core_curriculum

Ann Am Thorac Soc Vol 13, No 11, pp 2057-2059, Nov 2016

Copyright (C) 2016 by the American Thoracic Society

DOI: 10.1513/AnnalsATS.201607-575CME

Internet address: www.atsjournals.org 
antibiotics had resolution of cough (3). However, only $16 \%$ of those who did not receive antibiotics had resolution of cough.

The results of these studies suggest that 20 to $30 \%$ of infants with persistent wheezing who undergo bronchoscopy with BAL will be found to have a lower airway infection that improves with appropriate antibiotic therapy. Use of BAL may also help minimize unnecessary antibiotic therapy and its risks of side effects, including rash and acquisition of resistance.

Recommendation 3: In infants with persistent wheezing despite treatment with bronchodilators, inhaled corticosteroids, or systemic corticosteroids, we recommend research studies that compare clinical outcomes among infants who are managed according to infant pulmonary function testing performed using the raised volume rapid thoracoabdominal compression (RVRTC) technique versus those who are managed according to clinical assessment alone.

Numerous studies have reported pulmonary function tests in children with wheezing, but most were in older children. Of the limited number of studies in infants, only two studies (from the same cohort of patients at different points in time) measured clinical outcomes after the assessment of bronchodilator responsiveness using the RVRTC technique $(4,5)$. Both studies found that bronchodilator responsiveness predicted acute episodes of wheezing necessitating treatment with systemic steroids. No studies compared clinical outcomes after management based on infant pulmonary function tests versus management based on clinical assessment alone.

The guideline committee decided there was insufficient evidence to judge the balance of benefits versus risks, burden, and cost of managing infants with wheezing according to the results of pulmonary function testing performed using the RVRTC technique. Benefits include raising or lowering the index of suspicion for various diseases such as interstitial lung disease and anatomic abnormalities, as well as guiding further diagnostic testing and management. Risks include sedation, gastric distension, aerophagia, and airway occlusion; in addition, testing requires significant resource utilization. The guideline committee recommended research studies to fill the knowledge gap.
Recommendation 4a: For infants without eczema who have persistent wheezing despite treatment with standard therapies, we suggest NOT using empiric food avoidance (conditional recommendation, very low quality of evidence).

Recommendation 4b: We recommend research to determine whether or not empiric food avoidance is beneficial for the subgroup of infants who are IgE-positive to food antigens.

In infants with persistent wheezing, but without eczema, four studies investigated the effect of empiric food avoidance on wheezing frequency. Three of the studies were randomized trials that assigned infants to receive either regular formula (standard diet) or a restricted diet (cow's milk-free or partially hydrolyzed formula) during the first 4 months of life. None of the trials found an effect on the incidence of wheezing. The fourth study was a prospective cohort study that evaluated the introduction of commonly allergenic foods before or after 6 months of age. The study found no effect on wheezing.

No studies investigated the subgroup of infants with positive IgE antigen testing to food, nor were there any that evaluated additional important outcomes such as healthcare utilization, medication usage, and parental distress.

Given that all studies to date have found no effect on wheezing and that food restriction is burdensome, the guideline committee recommended against the routine empiric avoidance of foods. The committee, however, acknowledge that a trial of food avoidance may be warranted in some patients, such as those with a history of respiratory symptoms triggered by food exposure or IgE positivity to specific food antigens. Additional research targeting such patients was recommended.

Recommendation 5: For infants with persistent wheezing that is not relieved by bronchodilators, inhaled corticosteroids, or systemic corticosteroids, we suggest 24-hour esophageal $\mathrm{pH}$ monitoring (conditional recommendation, very low quality of evidence).

No randomized trials were identified comparing clinical outcomes among patients who underwent 24-hour esophageal $\mathrm{pH}$ monitoring to detect gastroesophageal reflux. However, four small case series were identified that used 24-hour esophageal $\mathrm{pH}$ monitoring to diagnose reflux in wheezy infants and children. One study identified reflux in $76 \%$ of the children with asthma studied. After 3 months of proton pump inhibitor therapy, there was significant improvement in respiratory symptoms, bronchodilator and systemic steroid use, and exacerbation and hospitalization frequency (6). These results were similar to the other three case series, which identified gastroesophageal reflux in 47 to $100 \%$ of infants and children with persistent wheeze and found that more than $83 \%$ improved with medical or surgical treatment.

The guideline committee decided that 24-hour esophageal $\mathrm{pH}$ monitoring with treatment of those with confirmed gastroesophageal reflux is preferable to an empiric trial of anti-acid therapy. Testing is generally well tolerated; in contrast, empiric therapy may be inappropriate in up to onethird of patients, creating unnecessary cost, burden, and risk. Proton pump inhibitor therapy has been associated with an increased risk of pneumonia in older children. Furthermore, children whose reflux requires fundoplication are unlikely to respond to empiric anti-acid therapy, which may be misinterpreted as a lack of evidence of gastroesophageal reflux, and the patient therefore may never be offered potentially curative surgical therapy.

There was significant variability in the way $\mathrm{pH}$ monitoring was performed, including the threshold used to declare studies abnormal. In addition, many studies were performed without impedance monitoring, which may have underestimated the prevalence of nonacid reflux.

Recommendation 6: For infants with persistent wheezing that is not relieved by bronchodilators, inhaled corticosteroids, or systemic corticosteroids, we suggest 24-hour esophageal $\mathrm{pH}$ monitoring rather than upper gastrointestinal series (conditional recommendation, very low quality evidence).

Three studies evaluating the accuracy of upper gastrointestinal series for identification of gastroesophageal reflux in infants and children indicate that upper gastrointestinal series have a sensitivity of 68 to $79 \%$ and specificity of $82 \%$ compared with esophageal $\mathrm{pH}$ monitoring, which is considered the reference standard.

The guideline committee did not believe that the potential benefits of an 
upper gastrointestinal series (it is less invasive, shorter, and hospitalization is not required) outweigh the 21 to $32 \%$ risk of a false-negative result (i.e., a test result excluding gastroesophageal reflux in a patient who actually has reflux), the likely result of which is a missed or delayed opportunity to treat. An upper gastrointestinal series also requires radiation exposure and patient cooperation.

Recommendation 7: For infants with persistent wheezing that is not relieved by bronchodilators, inhaled corticosteroids, or systemic corticosteroids, we suggest 24-hour esophageal $\mathrm{pH}$ monitoring rather than gastrointestinal scintigraphy (conditional recommendation, very low quality evidence).

In the only study comparing scintigraphy to 24-hour esophageal $\mathrm{pH}$ monitoring, scintigraphy had a sensitivity and specificity of 15 and $73 \%$, respectively (7). The guideline committee did not believe that the potential benefits of gastroesophageal scintigraphy (less invasive, shorter testing) outweigh the $85 \%$ risk of a false-negative result, the likely result of which is a missed or delayed opportunity to treat. Scintigraphy also requires radiation and is expensive.

Recommendation 8: For infants without neurologic pathology with persistent wheezing that is not relieved by bronchodilators, inhaled corticosteroids, or systemic corticosteroids, we suggest a swallowing function study to evaluate for aspiration (weak recommendation, very low quality of evidence).

Two case series reported the prevalence of swallowing dysfunction and clinical outcomes among infants younger than 12 months of age with chronic respiratory symptoms. The series found swallowing dysfunction in 10 to $15 \%$ of patients who had respiratory symptoms/vomiting. The infants were treated with thickening of oral or tube feeds (nasogastric, gastrostomy, or nasojejunal feeds), which resulted in an improvement in respiratory symptoms in more than $90 \%$ of infants.

On the basis of this, the guideline committee concluded that a swallowing evaluation may lead to management that reduces symptoms, medication usage, further diagnostic testing, and health care utilization, as well as providing parental reassurance. These benefits were believed to outweigh the drawbacks, which include the cost, need for a speech pathologist, need for patient cooperation, risk of aspiration during the study, and radiation exposure.

\section{Limitations and Future Directions}

Given the prevalence of wheezing in infants, there is an urgent need for large clinical studies that address the safety, efficacy, and cost effectiveness of the diagnostic tests described in this guideline to be conducted to inform clinical decisions in this vulnerable population.

For qualifying remarks related to the summary above, see the full clinical practice guideline (2).

Author disclosures are available with the text of this article at www.atsjournals.org.

\section{References}

1 Ren CL, Esther CR, Debley J, Sockrider M, Yilmaz O, Amin N, BazzyAssad A, Davis SD, Durand M, Ewig JM, et al. Official ATS clinical practice guidelines: diagnostic evaluation of infants with recurrent or persistent wheezing. Am J Respir Crit Care Med 2016;194: 356-373.

2 Martinez FD, Wright AL, Taussig LM, Holberg CJ, Halonen M, Morgan WJ; The Group Health Medical Associates. Asthma and wheezing in the first six years of life. N Engl J Med 1995;332:133-138.

3 Marchant J, Masters IB, Champion A, Petsky H, Chang AB. Randomised controlled trial of amoxycillin clavulanate in children with chronic wet cough. Thorax 2012;67:689-693.
4 Debley JS, Stamey DC, Cochrane ES, Gama KL, Redding GJ. Exhaled nitric oxide, lung function, and exacerbations in wheezy infants and toddlers. J Allergy Clin Immunol 2010;125:1228-1234.e13.

5 Elliott M, Heltshe SL, Stamey DC, Cochrane ES, Redding GJ, Debley JS. Exhaled nitric oxide predicts persistence of wheezing, exacerbations, and decline in lung function in wheezy infants and toddlers. Clin Exp Allergy 2013;43:1351-1361.

6 Yüksel H, Yilmaz O, Kirmaz C, Aydoğdu S, Kasirga E. Frequency of gastroesophageal reflux disease in nonatopic children with asthmalike airway disease. Respir Med 2006;100:393-398.

7 Balson BM, Kravitz EK, McGeady SJ. Diagnosis and treatment of gastroesophageal reflux in children and adolescents with severe asthma. Ann Allergy Asthma Immunol 1998;81:159-164. 